\title{
Neurocisticercosis: recomendaciones de tratamiento a propósito de tres casos
}

\author{
E. ÁLVAREZ-RODRÍGUEZ, R. TORRES-GÁRATE, A. GUTIÉRREZ LARRÁINZAR, \\ J. CABELLO, D. ESPINÓS PÉREZ
}

Servicio de Medicina Interna I. Hospital Universitario Clínico San Carlos. Madrid NEUROCYSTICERCOSIS: REPORT OF THREE CASES AND RECOM-
MENDATIONS OF TREATMENT

\section{RESUMEN}

La neurocisticercosis es una patología que nos estamos encontrando actualmente en la población inmigrante. La produce el parásito Taenia solium cuando se enquista en el sistema nervioso central. La clínica más frecuente es la crisis focal aunque se puede presentar como crisis generalizada; pueden aparecer también hidrocefalia, hipertensión intracraneal e incluso encefalitis. Su diagnóstico se basa en estudios de neuroimagen fundamentalmente. El tratamiento aún es controvertido y se ha de individualizar. La indicación de antiparasitarios como el albendazol y el prazicuantel ha de considerarse según la viabilidad del parásito, el número de lesiones y su localización. Si un quiste crece siempre está indicado su tratamiento. En el caso de aparecer hipertensión intracraneal, ésta debe ser tratada antes de tomar ninguna otra medida contra la enfermedad parasitaria. Los antiepilépticos son necesarios para tratar los síntomas. En ocasiones también está recomendado el uso de esteroides antes y durante la administración de antiparasitarios e incluso de forma individual.

\begin{abstract}
Neurocysticercosis is a pathology that we are finding nowadays over the immigrant population. It is produced by the Taenia solium parasite when it encyst into the central nervous system. The most frequent clinic is the focal crisis though it can come up as a general crisis; hydrocephalus, intracranial hypertension and even encephalitis can also happen. Its diagnosis is mainly based on imaging methods. The treatment is still controversial and must be individualized. The indication of antiparasitic drugs like albendazole and praziquantel has to be considered depending on the viability of the parasite, the number of cysts and their localization. If a cyst grows up the treatment is always recommended. In the case of an intracranial hypertension arises, this has to be treated before initiating any other action against the parasitic disease. Antiepileptic drugs are necessary to treat the symptoms. In some cases it is also recommended the use of steroids before and during the administration of antiparasitic therapy and even independently on their own.
\end{abstract}

KEY WORDS: Cysticercosis. Neurocysticercosis. Cestode. Taenia solium. Epilepsy. Antiparasitic drugs. Albendazole. Praziquantel. nia solium. Epilepsia. Antiparasitarios. Albendazol. Prazicuantel.

Álvarez-Rodríguez E, Torres-Gárate R, Gutiérrez Larraínzar A, Cabello J, Espinós Pérez. D. Neurocisticercosis: recomendaciones de tratamiento a propósito de tres casos. An Med Interna (Madrid) 2004; 21: 382-386.

\section{INTRODUCCIÓN}

La cisticercosis es la enfermedad parasitaria más frecuente del sistema nervioso central (SNC) (1-3). De hecho, en países endémicos, sobre todo América latina, África y Asia, se considera la primera causa de epilepsia por delante de tumores cerebrales, anomalías vasculares y traumatismos. En los países europeos como en España, no es una enfermedad endémica pero se ven casos incidentes con cada vez más frecuencia en la población inmigrante, lo que hace necesaria alguna puntualización sobre la patología, sobre todo en lo que concierne al tratamiento, ya que existe una gran confusión en la literatura (en su mayoría latinoamericana).

\section{CASOS APORTADOS}

Caso 1. Varón ecuatoriano de 27 años que había presentado tres años atrás una primera crisis convulsiva en su país, siendo diagnosticado de neurocisticercosis. Fue tratado con albendazol $600 \mathrm{mg} /$ día durante 48 días y con antiepilépticos quedando asintomático. Comienza ahora con nuevas crisis consistentes en pérdida de fuerza en brazo derecho por lo que se cambia su medicación anticonvulsivante y se realiza resonancia magnética nuclear (RM) cerebral en busca de actividad de la enfermedad. Se observan cinco lesiones: tres hipointensas en $\mathrm{T}_{2}$ en relación con calcificación y con realce anular tras la administración de contraste paramagnético, otra lesión sin calcificar y otra en asta occipital del ventrículo lateral izquierdo sin calcificación ni realce con contraste. No se observan signos de hidroce-

Trabajo aceptado: 11 de marzo de 2004 
falia. El paciente se encuentra sin focalidad neurológica en la exploración y con buen aspecto general.

Caso 2. Varón de 20 años de nacionalidad ecuatoriana que, a los 7 meses de residir en España, presenta una crisis convulsiva generalizada. En la RM cerebral se descubre una lesión nodular de $1 \mathrm{~cm}$ en corteza occipital izquierda con una zona central hiperintensa en $\mathrm{T}_{2} \mathrm{y}$ edema perilesional con realce en anillo, todo ello típico de neurocisticercosis. El paciente presenta una exploración física y neurológica normal.

Caso 3. Mujer ecuatoriana de 29 años que a los pocos meses de estar en España debuta con tres episodios de crisis convulsiva generalizada. Se le realiza RM cerebral en la que se encuentra una lesión en lóbulo parietal izquierdo con realce en anillo muy sugerente de neurocisticercosis. La exploración física y neurológica es normal.

Evolución: en los tres casos, se mantuvo el antiepiléptico y se inició tratamiento con albendazol $15 \mathrm{mg} / \mathrm{kg} /$ día durante una semana, prazicuantel $100 \mathrm{mg} / \mathrm{kg}$ en dosis única, dexametasona y cimetidina. Los tres desarrollaron leucocitosis con desviación izquierda e hiperglucemia con el tratamiento esteroideo. El resto de parámetros analíticos y bioquímicos fueron normales. No presentaron más crisis epilépticas.

\section{DISCUSIÓN}

El parásito: la enfermedad está producida por el cestodo Taenia solium. El gusano adulto vive en el intestino delgado del hombre. Está compuesto por una cabeza o escólex con órganos de fijación y varios anillos o proglótides que liberan huevos fertilizados a las heces. El huésped intermediario natural es el cerdo que ingiere estos huevos, se liberan las larvas en su estómago y éstas pasan vía hematógena hacia tejidos propicios (habitualmente músculo estriado, ojos y SNC) donde se enquistan. Los hombres desarrollan cisticercosis por la ingesta accidental de los huevos fertilizados mediante contaminación feco-oral (Fig. 1). Otro mecanismo de transmisión poco frecuente y no totalmente aceptado es la autoinfestación. Consiste en el paso mediante movimientos antiperistálticos de huevos fertilizados desde el intestino delgado humano infectado con la teniasis hacia el estomago, donde se liberarían las larvas.
Clínica de la neurocisticercosis: la sintomatología de la cisticercosis cerebral depende de la localización de la lesión. En lesiones parenquimatosas suele debutar con crisis epilépticas e incluso con síntomas focales debidos a un efecto de masa. La inflamación que los rodea puede ser causa de infartos cerebrales al ocluir pequeñas arterias perforantes (1). Las lesiones extraparenquimatosas pueden causar hidrocefalia, bien por obstrucción mecánica del sistema ventricular, o bien por aracnoiditis al localizarse en el espacio subaracnoideo. En muchos casos, los síntomas aparecen años después de la invasión del SNC por la inflamación, el efecto de masa o por calcificaciones residuales. De hecho, cuando los cisticercos están ya muertos o han degenerado, habitualmente calcifican e incluso forman una inflamación granulomatosa a su alrededor que puede ser igualmente la causa de muchos de los síntomas de los pacientes. Una manifestación grave pero poco frecuente es la encefalitis, cuando existen múltiples lesiones con una importante reacción inflamatoria.

Diagnóstico de la neurocisticercosis: el diagnóstico se basa en estudios de neuroimagen, datos clínicos, y sobre todo datos epidemiológicos. En los análisis rutinarios ocasionalmente podemos encontrar eosinofilia. Los huevos del parásito característicamente no se encuentran en las heces ya que el huésped intermediario no tiene habitualmente $T$. solium viable en su intestino. En cuanto a las técnicas de imagen, la resonancia magnética nuclear (RM) es más sensible que la tomografía axial computarizada (CT) para pequeñas lesiones y también para las intraventriculares o medulares, pero la CT, además de más barata, detecta mejor pequeñas áreas de calcificación. El parásito pasa por distintas fases. Al principio podemos observar una vesícula con un contenido líquido de intensidad de señal similar a la del líquido cefalorraquídeo (LCR). La visualización de un nódulo mural de alta densidad correspondería al escólex y es patognomónico de la neurocisticercosis activa $(2,4,5)$. El cisticerco vivo produce sólo una leve reacción inflamatoria dando pocos síntomas. Cuando se produce su muerte, bien espontáneamente, bien tras el tratamiento o bien gracias a la respuesta inmune del huésped, se

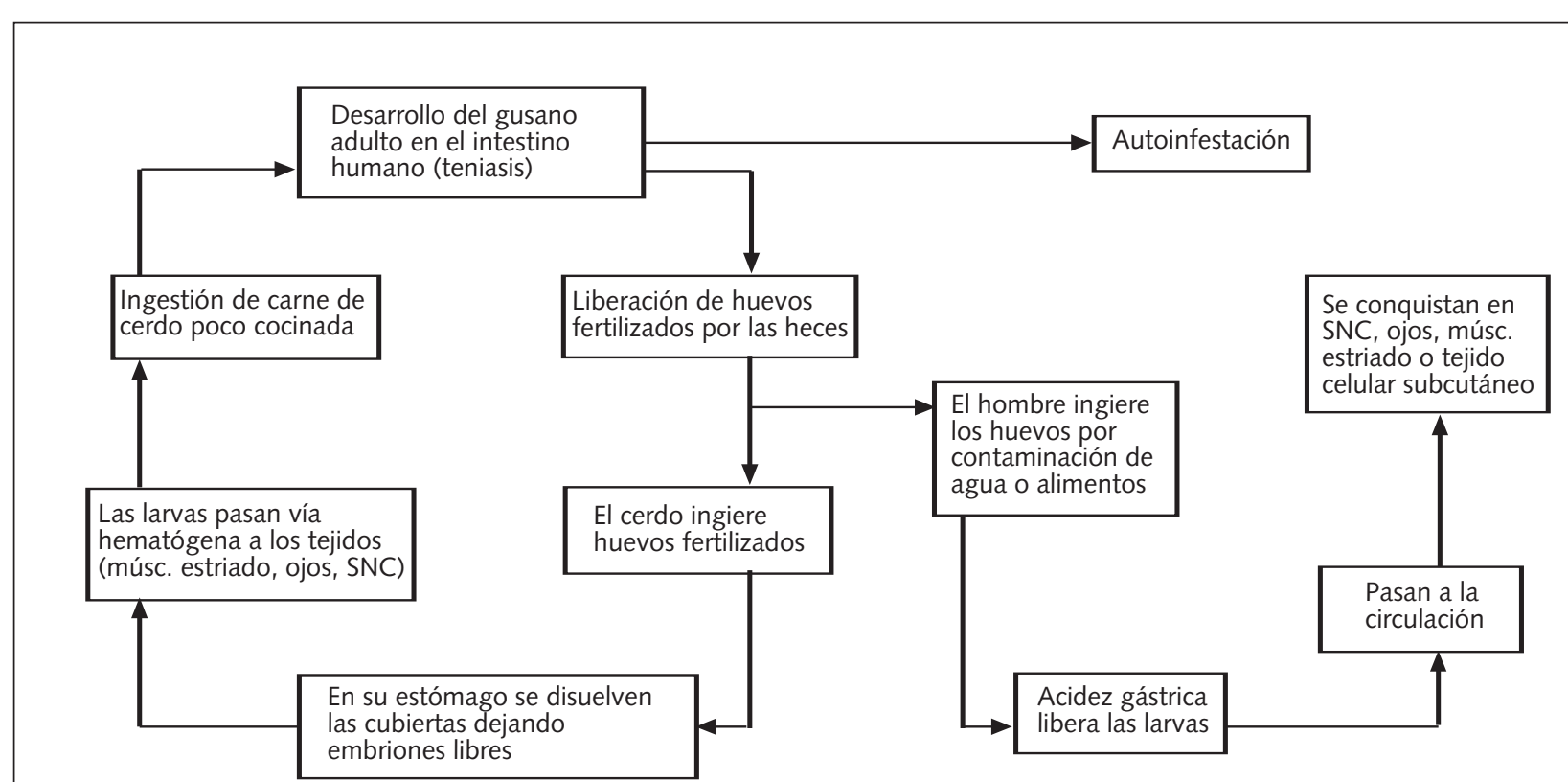

Fig. 1. Ciclo de la Taenia solium. 


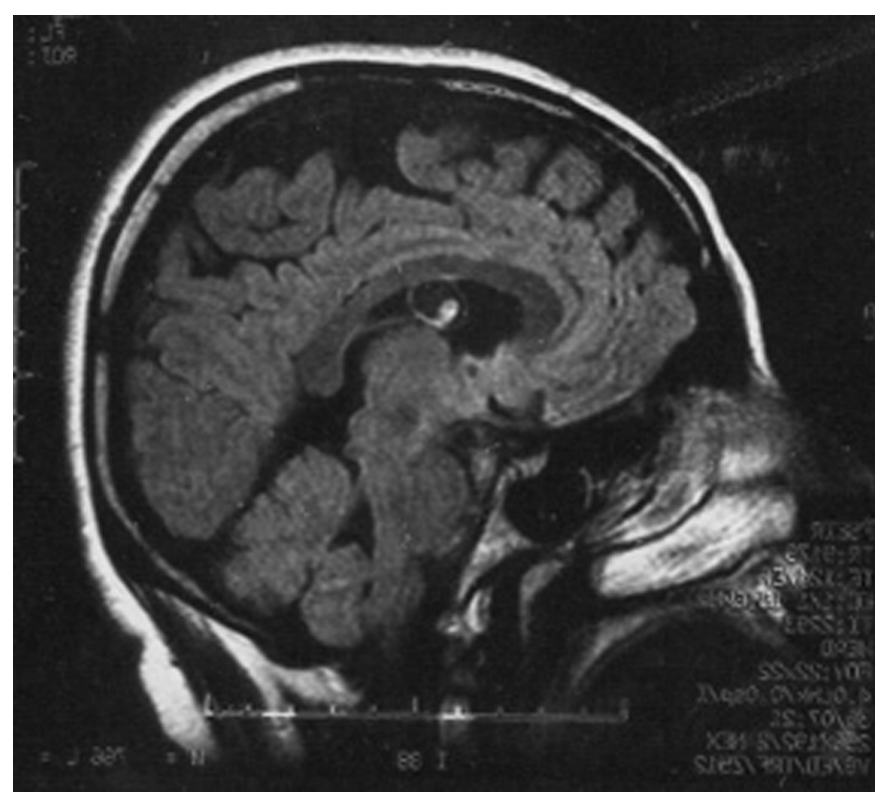

Fig. 2.

produce una intensa reacción inflamatoria alrededor apareciendo un realce en anillo tras la administración de contraste. Meses más tarde degenera, se fibrosa y puede calcificarse (2). El análisis del LCR suele estar alterado cuando la enfermedad está activa. Puede aparecer pleocitosis mononuclear, aumento de proteínas y glucosa normal o disminuida si los cisticercos se localizan cerca o en los ventrículos cerebrales $(1,4)$. Por otra parte, mediante el método ELISA pueden encontrarse anticuerpos para cisticercosis en sangre y LCR. Es el método más empleado aunque su sensibilidad es menor que el Western Blot y existen reacciones cruzadas con antígenos de otros helmintos. La posibilidad de serologías negativas suele ser mayor cuanto menor número de lesiones aparecen en las pruebas de imagen $(4,6)$.
Armas terapéuticas contra la neurocisticercosis: existen distintos aspectos dentro del tratamiento de una cisticercosis cerebral. Por un lado el tratamiento antiparasitario y, por otro, el tratamiento de los síntomas. Además, existen complicaciones que pueden requerir la cirugía.

Contamos con antiparasitarios como el albendazol y el prazicuantel. Clásicamente el tratamiento de la neurocisticercosis ha consistido en la administración de albendazol 15 $\mathrm{mg} / \mathrm{kg} /$ día separado en dos tomas durante 28 días (aunque hay autores que defienden terapias de sólo 8 días y hasta de varios meses). Y cuando se utiliza el prazicuantel se hace a dosis de $50 \mathrm{mg} / \mathrm{kg} /$ día en tres tomas durante 15 días, siendo el albendazol más eficaz en algún estudio $(3,4)$. Actualmente parece que la administración de albendazol $15 \mathrm{mg} / \mathrm{kg} /$ día durante una semana y prazicuantel $100 \mathrm{mg} / \mathrm{kg}$ en dosis única es igual o más eficaz que las terapias clásicas $(1,3)$. Se puede asociar además dexametasona de 6 a $12 \mathrm{mg} /$ día para disminuir la reacción inflamatoria que resulta de la destrucción de los quistes y que podría afectar a la pared de los vasos con el consiguiente riesgo de infarto $(1,4)$. Debemos tener en cuenta además que la dexametasona puede aumentar los niveles de albendazol en plasma (4) y sin embargo puede disminuir los de prazicuantel $(3,4)$. Para obtener mayores niveles plasmáticos de albendazol se puede utilizar la cimetidina en dosis de $400 \mathrm{mg}$ dos veces al día (4).

Otros síntomas de neurocisticercosis han de ser manejados individualmente. La epilepsia con anticonvulsivantes; la hidrocefalia con derivación ventricular antes del tratamiento con albendazol o prazicuantel; la encefalitis con esteroides y diuréticos osmóticos, antes también de los antiparasitarios.

Indicaciones de tratamiento: sin embargo, el empleo de antiparasitarios es controvertido. Algunos estudios apuntan circunstancias concretas en las que estas drogas pueden no tener beneficio alguno frente a la abstención del tratamiento o frente a la administración de esteroides únicamente; e incluso situaciones en las que tratar con fármacos antiparasitarios puede ser perjudicial para el paciente. El que el tratamiento de

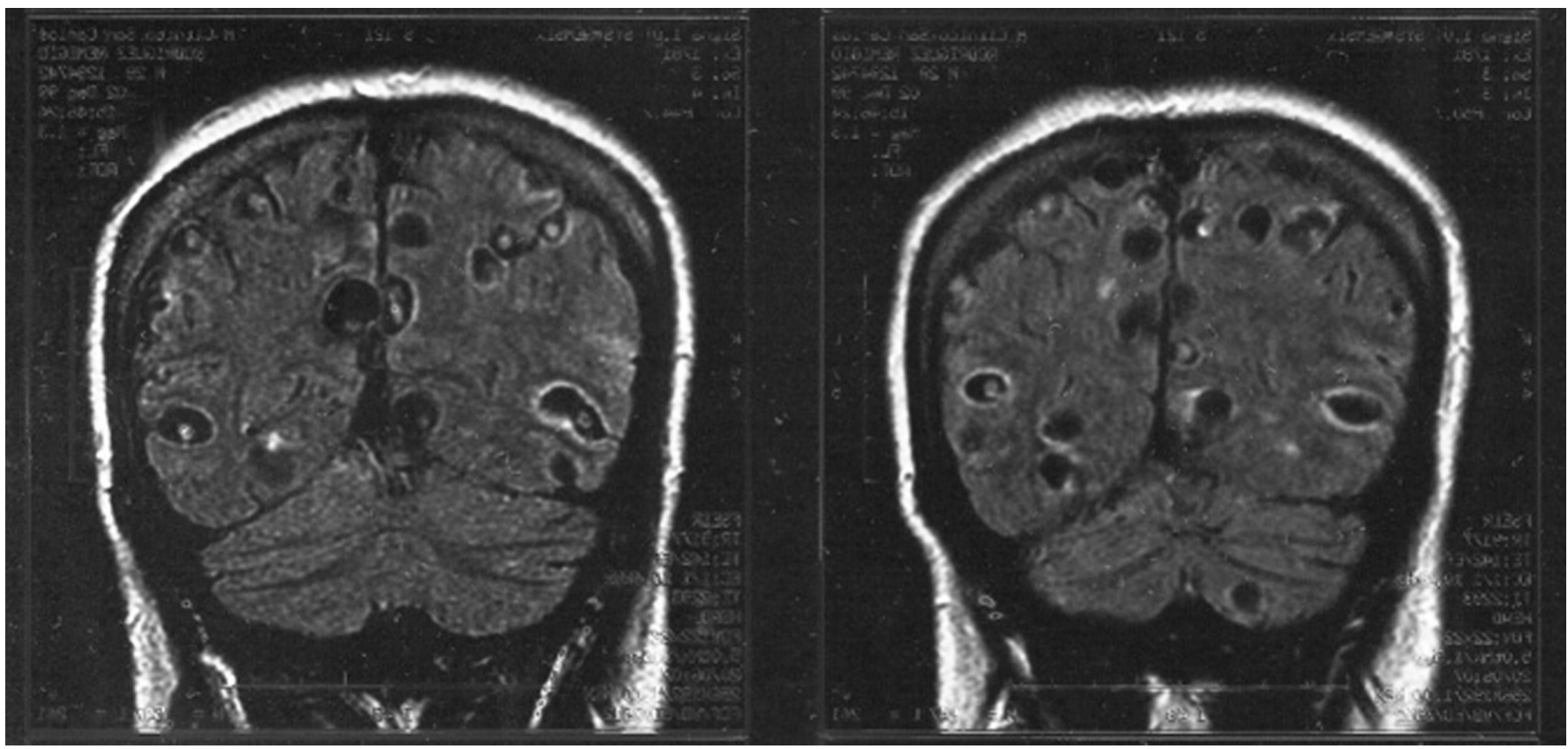

Fig. 3. 
TABLA I

GUÍAS PARA EL USO DE ANTIPARASITARIOS EN NEUROCISTICERCOSIS

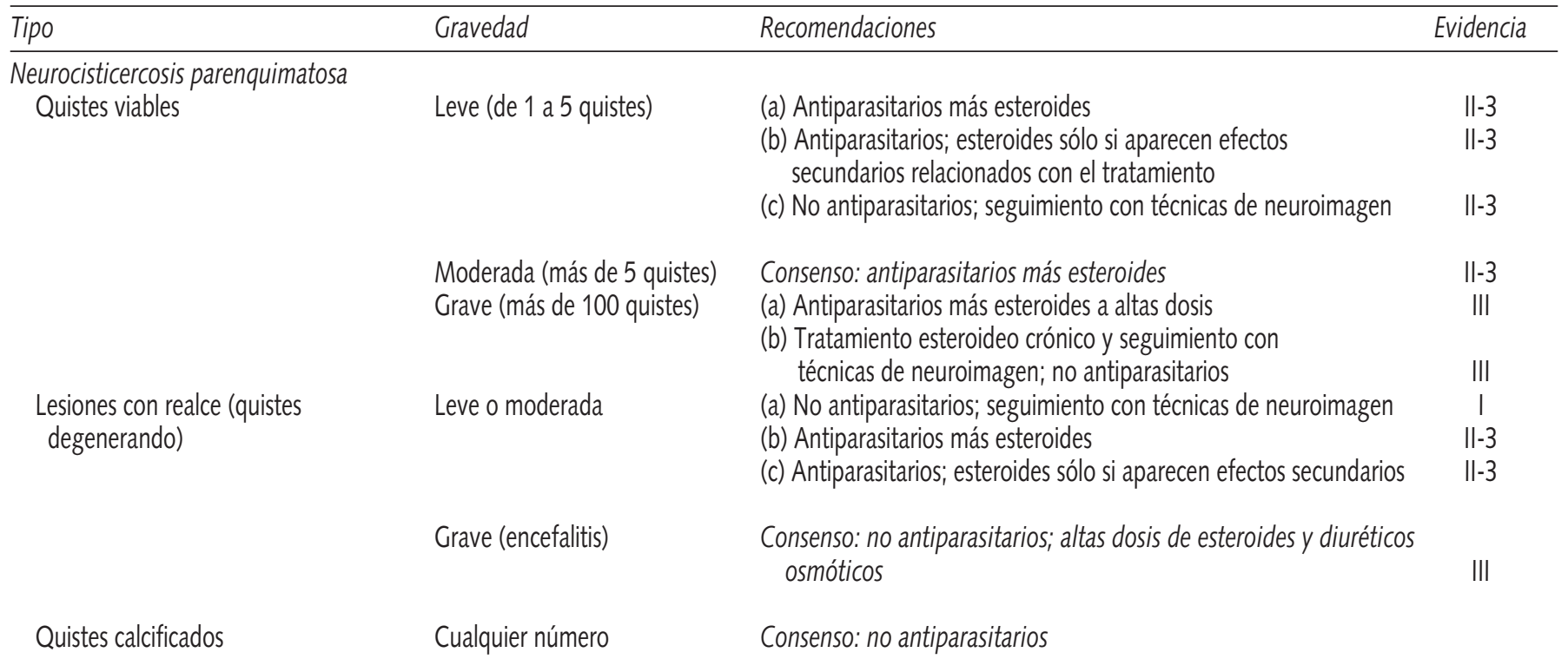

Neurocisticercosis extraparenquimatosa

Cisticercosis ventricular

Quistes subaracnoideos, quistes

gigantes o en crecimiento y meningitis crónica
Consenso: extirpación neuroendoscópica. Si no está disponible:

(a) Derivación ventricular seguido de antiparasitarios más esteroides

(b) Cirugía abierta

Consenso: antiparasitarios con esteroides, derivación ventricular si hay hidrocefalia
III

III

III

$\|-3$

Consenso: derivación ventricular; no antiparasitarios

III

Consenso: cirugía; algún buen resultado anecdótico con

albendazol y esteroides

Consenso: extirpación quirúrgica (2)

Cisticercosis oftalmológica (1)

Traducida de Héctor H García et al. Current consensus guidelines for treatment of neurocysticercosis. Clin Microbiol Rev 2002; 15 : 753.

( $a$, b y c) son niveles de recomendación

I evidencia obtenida en al menos un ensayo controlado prospectivo y randomizado

II-1 evidencia obtenida desde ensayos controlados bien diseñados pero sin randomizar

II-2 evidencia obtenida desde cohortes bien diseñadas o estudios de casos-controles, preferentemente por más de un centro o grupo de investigación

II-3 evidencia obtenida desde múltiples series con o sin intervención, incluyendo malos resultados de experimentos no controlados

III opiniones de autoridades basadas en la experiencia clínica; estudios descriptivos y casos publicados; publicaciones de comités de expertos

(1) Dada la rareza de estas presentaciones, el tratamiento fue discutido en base a lo publicado

(2) El uso de albendazol con metilprednisolona para el tratamiento de la cisticercosis retiniana de forma preoperatoria ha sido publicado pero aun no discutido

la neurocisticercosis no esté estandarizado es debido a que es una enfermedad con múltiples presentaciones y clínicas que requieren un tratamiento específico, y a que existe una gran confusión entre los planteamientos de los diversos estudios y sus resultados. Recientemente, en un esfuerzo por esclarecer estas dudas, se han establecido unas guías con las recomendaciones propuestas por los expertos y basadas en el análisis de la literatura (3). Se presentaron unas nociones básicas en las que hubo total consenso: a) el tratamiento debe individualizarse según el número de lesiones y su localización, así como según la viabilidad del parásito; b) el crecimiento de un quiste parenquimatoso no es muy habitual, pero de darse el caso, es una situación que precisa tratamiento urgente (ya sea mediante antiparasitarios o cirugía); c) cuando aparece hipertensión intracraneal secundaria a neurocisticercosis, es prioritario el tratamiento de ésta antes de plantearse ninguna otra medida contra la enfermedad parasitaria; d) los antiepilépticos son los fármacos de elección para el tratamiento de las crisis si aparecieran, y se utilizan de forma semejante a cuando se usan para cualquier otra enfermedad que produzca esta sintomatología a través de un foco orgánico. Los antiparasitarios no deben usarse como alternativa a los antiepilépticos. En cuanto a las situaciones en que están indicados los antiparasitarios, se resumieron en la tabla I (3). 


\section{Bibliografía}

1. Del Brutto OH. Cerebrovascular disease in the tropics. Rev Neurol 2001; 33: 750-762.

2. Martínez HR, Rangel-Guerra R, Arredondo-Estrada JM, Marfil A, Onofre J. Medical and surgical treatment in neurocysticercosis: a magnetic resonance study of 161 cases. J Neurol Sci 1995; 130: 25-34.

3. García HH, Evans CAW, Nash TE et al. Current consensus guidelines for treatment of neurocysticercosis. Clin Microbiol Rev 2002; 15: 747-756.

4. Coyle CM, Wittner M, Tanowitz HB. Cysticercosis. En: Guerrant RL, Walker DM, Weller PF, eds. Essentials of tropical infectious diseases. Philadelphia: Churchill Livingstone, 2001; 472-476.

5. Chang KH, Han MH. MRI of CNS parasitic disease. J Magn Reson Imaging 1998; 8: 297-307.

6. García HH, Parkhouse RM, Gillman RH et al. Serum antigen detection in the diagnosis, treatment, and follow-up of neucysticercosis patients.
Trans R Soc Trop Med Hyg 2000; 94: 673-676.

7. Del Brutto OH, Sotelo J. Neurocysticercosis: an update. Rev Infect Dis 1998; 10: 1075-1087.

8. García HH, González AE, Evans CA, Gilman RH. Cisticercosis working group in Peru. Lancet 2003; 362: 547-556.

9. Carpio A. Neurocysticercosis: an update. Lancet Infect Dis 2002; 2(12): 751-762.

10. Del Brutto OH, Rajshekhar V, White AC et al. Proponed diagnostic criteria for neurocysticercosis. Neurology 2001; 57(2): 177-183.

11. Pérez-López C, Isla-Guerrero A, Álvarez F et al. Update in neurocysticercosis treatment. Rev Neurol 2003; 36(9): 805-811.

12. Font C, Ruiz JA, Muñoz C, Pardós F, Corachan M. Neurocisticercosis en España. A propósito de 4 casos observados en pacientes inmigrantes de países endémicos. An Med Interna (Madrid) 1999; 16: 89-91. 